\title{
ÍNDICE DE IMPACTO DOS RESÍDUOS SÓLIDOS URBANOS NA SAÚDE PÚBLICA (IIRSP): METODOLOGIA E APLICAÇÃO
}

\author{
IMPACT INDEX OF SOLID WASTES MANAGEMENT SYSTEMS ON PUBLIC HEALTH \\ (IIRSP): METHODOLOGY AND APPLICATION
}

ANA BEATRIS SOUZA DE DEUS

Doutora em Recursos Hídricos e Saneamento Ambiental, Prof. da Universidade de Caxias do Sul/Instituto de Saneamento Ambiental

\author{
SÉRGIO JOÃO DE LUCA
}

PhD em Engenharia Ambiental. Prof. da Universidade Federal do Rio Grande do Sul/Instituto de Pesquisas Hidráulicas

\section{Robin Thomas CLARKE}

D.Sc. Hidrólogo, Prof. Visitante da Universidade Federal do Rio Grande do Sul/Instituto de Pesquisas Hidráulicas

Recebido: 09/04/04 Aceito: 20/08/04

\section{RESUMO}

A redução do número de enfermidades produzidas, direta ou indiretamente, pelos resíduos sólidos depende de uma coleta eficiente e de uma adequada disposição final. Com as informaçôes obtidas da aplicação de um questionário enviado aos municípios sul rio-grandenses divididos em sete regiōes homogêneas, foram desenvolvidos indicadores e, por meio de procedimentos estatísticos não paramétricos, estruturados oito índices (índices específicos) que formaram um índice geral de controle de qualidade dos serviços de limpeza urbana. $\mathrm{O}$ artigo apresenta um sumário e um exemplo da metodologia utilizada para a criação do Índice de Impacto dos Resíduos Sólidos Urbanos na Saúde Pública (IIRSP), o qual mais diretamente espelha a relação Resíduos sólidos-homem-saúde pública.

PALAVRAS-CHAVE: Resíduos sólidos, indicadores, índice de impacto dos resíduos sólidos urbanos na saúde pública (IIRSP).

\begin{abstract}
The reduction in the number of diseases produced directly or indirectly by municipal solid waste depends on an efficient collection and appropriate final disposal. With the information obtained from a questionnaire sent to the Rio Grande do Sul municipalities, divided into seven homogeneous regions, indicators were developed and, by means of non-parametric statistical procedures, eight index were structured (specific indices), which form a general quality control index for solid wastes services. In this paper is presented a summary and an example of the methodology used to create the Impact Index of Solid Waste Management Systems on Public Health (IIRSP), which more directly reflects the solid waste-man-public health relationship.
\end{abstract}

KEYWORDS: Solid wastes, indicators, impact index of solid waste management systems on public health (IIRSP).

\section{INTRODUÇÃO}

Entre os diversos problemas ambientais existentes, o dos resíduos sólidos urbanos tem-se tornado um dos maiores desafios da atualidade. Com o crescimento acelerado da população, houve incremento na produção de bens e serviços. Estes, por sua vez, à medida que são produzidos e consumidos, acarretam uma geração cada vez maior de resíduos, os quais, coletados ou dispostos inadequadamente, trazem significativos impactos à saúde pública e ao meio ambiente.

No Brasil e, especificamente, no Estado do Rio Grande do Sul, os serviços de limpeza urbana são atribuiçôes das administraçōes municipais. A maioria destas desconhecem as variáveis ambien- tais correlacionadas com os resíduos sólidos urbanos, importantes para administrar com eficiência e eficácia o setor. Sendo assim, propóe-se um conjunto de indicadores, estruturados e sistematizados, ligados à geração de resíduos sólidos urbanos que serão a base para a estruturação e formulação de um índice que relaciona resíduos sólidos urbanos, doenças e o ser humano, com o intuito de permitir uma hierarquização de atitudes e investimentos dentro da área de gerenciamento de resíduos sólidos urbanos.

Conforme Deus (2000) diversos autores relacionam casos de doenças aos resíduos sólidos. Entretanto, a influência dos resíduos urbanos se faz sentir, principalmente, por vias indiretas, ou seja, a conexão Resíduos Sólidos-Vetor-Homem explica as trajetórias pelas quais pode ocorrer a transmissão de doenças oriundas da coleta e/ou disposição inadequada daqueles.

Os sistemas de limpeza urbana (SLUs), cujo estabelecimento é uma necessidade e decorrência das atividades desenvolvidas pelo homem em sua comunidade, possuem as seguintes atribuiçōes:

- Coleta: principal atividade, corresponde à remoção dos resíduos sólidos produzidos na área urbana dos municípios. A coleta e o transporte para o local de tratamento/disposição final são ações de grande visibilidade para a população, tendo como objetivo impedir o desenvolvimento de vetores transmissores de doenças, os quais encontram alimento e abrigo nos resíduos;

- Limpeza de ruas e logradouros públicos: se- 
gunda atribuição em importância para a população, tem por objetivos manter a limpeza das cidades e áreas urbanas, prevenir enchentes e o assoreamento de rios e arroios e minimizar os riscos à saúde;

- Transporte: é uma atividade distinta da coleta quando as distâncias de remoção até os pontos de destinação final passam a ser longos;

- Tratamento/disposição final: geralmente, esta operação é efetuada imediatamente após a coleta. Quando o processamento tem por objetivo fundamental a diminuição dos inconvenientes sanitários ao homem e ao meio ambiente e o reaproveitamento de materiais e energia, dizse então que os resíduos sólidos foram submetidos a um tratamento. A disposição final corresponde a última fase do sistema; - Serviços complementares: podem ser citados, a limpeza do sistema de captação de águas pluviais, monumentos, túneis, escadarias, abrigos e feiras, a capina, entre outros.

Segundo UNEP (1995), os indicadores podem transformar-se em uma importante ferramenta para tornar acessível a informação científica e técnica para os diferentes grupos de usuários. A função dos indicadores é resumir a grande quantidade de dados, tornando acessível o seu entendimento.

Os índices são parâmetros que medem cada indicador, atribuindo-lhe valores numéricos (Adriaanse, 1993) ou, de acordo com Bakkes et al. (1994), são resultados da combinação de várias variáveis ou parâmetros em um só valor, assumindo um peso relativo a cada componente do índice. Eles permitem observar e acompanhar a situação do meio ambiente, o impacto e as conseqüências dos processos de desenvolvimento sobre os recursos naturais, as funçôes ecológicas e as inter-relações entre os diferentes fatores do desenvolvimento. O sub-índice é um passo intermediário entre o indicador e o índice. Ele serve para agrupar os diversos indicadores facilitando a construção do índice.

A adoção de indicadores, sub-índices e índices podem servir como referencial para o planejamento e melhoria operacional dos serviços de limpeza urbana nos municípios, pois demonstram a influência dos sistemas na área da saúde pública, no desenvolvimento econômico, social e ambiental regional; mostram, por meio de diagnósticos, as necessidades de desenvolvimento científico e tecnológico do setor; e facilitam as definiçôes dos objetivos e das metas para futuros empreendimentos, levando em consideração no seu projeto, as preocupaçôes de proteção ambiental (Deus, 2000).

O critério de utilizar uma grande variedade de indicadores na avaliação dos serviços de limpeza urbana representa, sem dúvida, uma opção metodológica aceitável, porque permite enriquecer a análise. Entretanto, introduz dificuldades para a comparação entre os diferentes municípios. Quando se comparam dois municípios que apresentam indicadores melhores e outros piores, não há como dizer, inequivocamente, qual deles apresenta um sistema com mais qualidade e eficiência que outro. Entretanto, os índices permitem dirimir essa dúvida.

A construção de um índice visa, em princípio, permitir a comparação entre indicadores agrupados comparando a mesma qualificação entre municípios, onde são considerados diferentes aspectos de suas respectivas realidades.

O objetivo deste artigo parece inédito, pois na literatura especializada não foram encontradas abordagens ou índices, correlacionando estatisticamente a eficiência dos serviços de limpeza urbana com efeitos na saúde pública.

\section{FUNDAMENTACÃO METODOLÓGICA}

O Estado do Rio Grande do Sul apresenta diferenças regionais internas devido à distribuição populacional, econômica e social. Essas diferenças influem diretamente nos resíduos sólidos gerados e, consequentemente, nas características dos serviços de limpeza urbana. Devido a isto, foram adotados dois critérios de coleta de informaçôes: um baseado na urbanização e outro na regionalização, pois municípios com população menor apresentam características totalmente diferentes daquelas encontradas em municípios com população muito maior. Além disso, não se pode comparar um município de uma região industrializada com outra, cuja atividade predominante seja, por exemplo, a agricultura. Estes critérios de urbanização e regionalização já foram empregados em outros trabalhos podendo ser citados De Luca (1999) e Oliveira et al. (1998). A seguir estão descritos os critérios empregados:

a) Critério de Urbanização-definição de faixas populacionais, tendo a finalidade de agrupar os municípios do estado do Rio Grande do Sul em função da população urbana, sendo estas:
- Faixa A: Municípios de pequeno porte, população urbana menor que 10.000 hab.;

- Faixa B: Municípios de médio porte, população urbana entre $10.000 \mathrm{e}$ 100.000 hab.;

- Faixa C: Municípios de grande porte, população urbana maior que 100.000 hab..

b) Critério de Regionalização - os municípios do estado do Rio Grande do Sul foram agrupados por regiōes, conforme a atividade econômica neles predominantes, sendo:

- Regiāo Nordeste: industrializada, possui grandes aglomerações urbanas e atividades agrícolas inexpressivas;

- Região Norte: agrícola, possui pequenas e médias propriedades; região muito heterogênea e pouco industrializada;

- Região Sul: pecuária e de grandes propriedades, possui crescimento econômico lento.

Seguindo estes critérios, o Estado do Rio Grande do Sul foi dividido em sete faixas de análise sendo estas: Região Nordeste/Faixa A, Regiāo Nordeste/Faixa B, Região Norte/Faixa A, Região Norte/Faixa B, Região Sul/Faixa A, Região Sul/Faixa B, Região Mista/Faixa C.

\section{Construção do índice relacionando os resíduos sólidos e a saúde pública}

Para a formulação do Índice de Impacto dos Resíduos Sólidos Urbanos na Saúde Pública (IIRSP) foram considerados todos os indicadores (variáveis) que, diretamente ou indiretamente, poderiam causar ou provocar danos à saúde humana e animal.

Por exemplo, a deficiência de coleta leva a população a procurar uma maneira de se livrar dos resíduos sólidos colocando-os em arroios, rios, terrenos baldios, entre outros, formando grandes monturos os quais tendem a atrair vetores. $\mathrm{O}$ mesmo ocorre com o déficit de tratamento e/ou disposição final, característica da maioria dos municípios do estado do Rio Grande do Sul. A falta de coleta e da disposição final adequada dos resíduos sólidos favorece a proliferação, por exemplo, de ratos, aves (urubus e garças), suínos, cachorros e gatos, destacadamente no local de disposição final. Sendo assim, existe a possibilidade de que os casos de cisticercose, leptospirose, teníase, toxoplasmose e triquinose estejam asso- 
ciadas a proliferação destes. Quanto menor o orçamento municipal destinado ao serviço de limpeza urbana maiores são as chances de ocorrerem doenças entre a população exposta a estes. Após análise criteriosa, o IIRSP proposto foi formado por oito indicadores, sendo eles:

- Indicador de déficit de coleta $\left(\mathrm{IND}_{14}\right)$;

- Indicador de déficit de tratamento/disposição final $\left(\mathrm{IND}_{41}\right)$;

- Indicador de cisticercose (IND)

- Indicador de leptospirose (IND 2$)$;

- Indicador de teníase (IND $)$;

- Indicador de toxoplasmose $\left(\mathrm{IND}_{4}\right)$;

- Indicador de triquinose (IND 5$)$; e

- Indicador do orçamento destinado aos SLUs $\left(\mathrm{IND}_{7}\right.$ ).

No processo de construção de um índice, a técnica estatística empregada deve ser capaz de reduzir os indicadores padronizados formados, agrupá-los e determinar qual é a participação (importância) de cada um. Para o desenvolvimento do índice específico foi utilizada a técnica desenvolvida por Kendall (Siegel, 1981). Esse é um procedimento de classificação não paramétrica, o qual consiste do cálculo de distâncias padronizadas de cada dado referente a um valor de controle. $\mathrm{O}$ fluxograma do procedimento de Kendall está esquematizado na Figura 1.

\section{Valores de controle}

Os valores de controle devem representar a situação ideal e a redução de impactos para o meio ambiente e ao homem. Como valor de controle (VC) foram adotados os valores 0 e 1 , conforme o indicador padronizado em questão. Por exemplo, o VC para os indicadores de saúde $\left(\mathrm{IND}_{1}, \mathrm{IND}_{2}, \mathrm{IND}_{3}, \mathrm{IND}_{4}, \mathrm{IND}_{5}\right)$ é igual a zero (0), pois é melhor não ter casos de doenças no município; para o indicador de orçamento destinado aos SLUs $\left(\mathrm{IND}_{7}\right)$ quanto maior a percentagem do orçamento destinado aos SLUs melhor, logo VC é igual a um (1); para os indicadores de déficit de coleta $\left(\operatorname{IND}_{14}\right)$ e de tratamento e disposição final (IND ${ }_{41}$ ) quanto menor o déficit, melhor para o meio ambiente, então VC será igual a zero (0).

\section{Padronização dos indicadores}

A agregação de indicadores para a formação de sub-índices exige, geralmente, que diferentes medidas sejam trans-

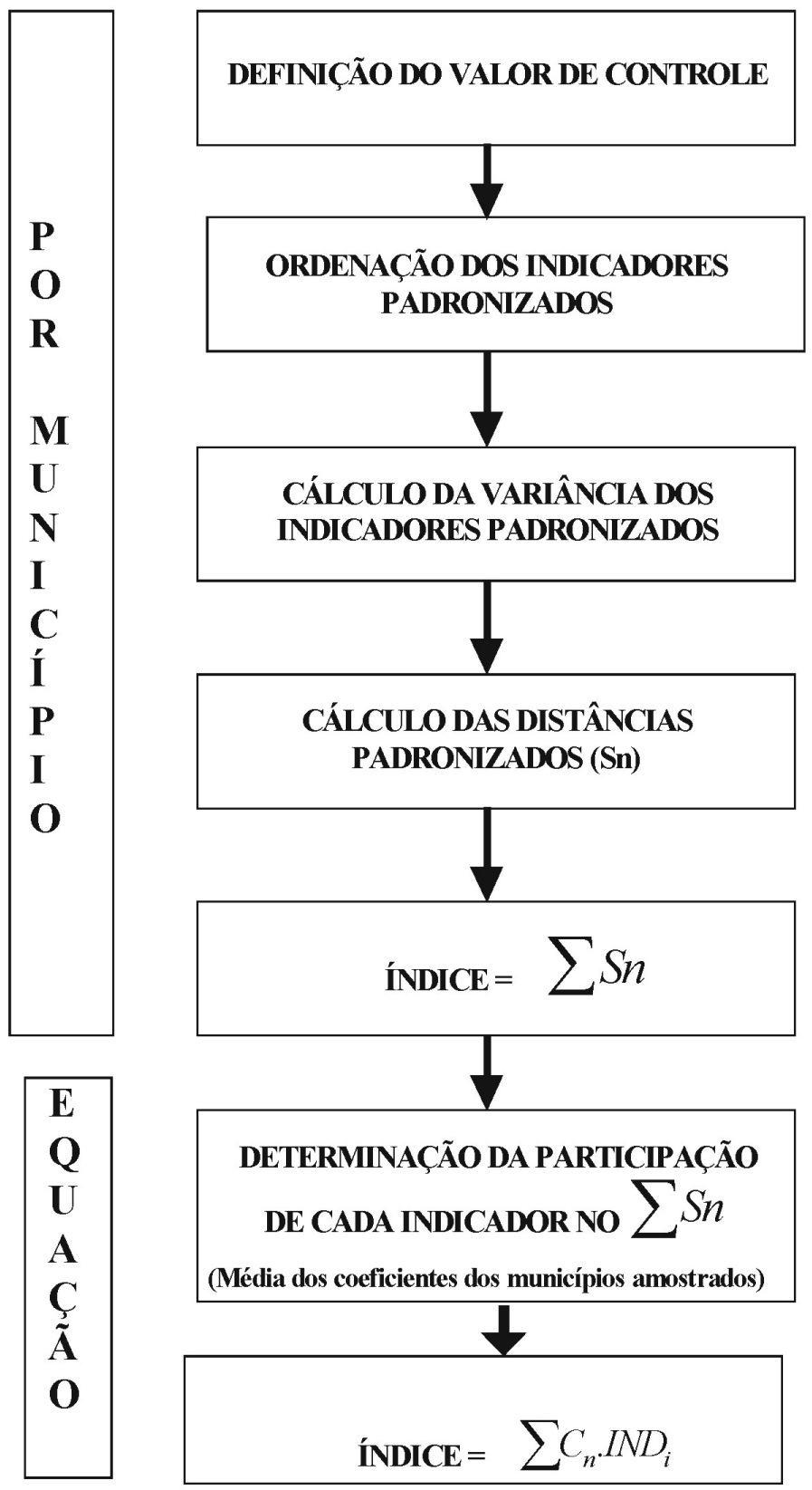

Figura I - Fluxograma do procedimento não paramétrico de Kendall para formular o Índice de Impacto dos Resíduos Sólidos Urbanos na saúde pública (adaptado de Siegel, 198I)

formadas em uma escala comum. A transformação em escala comum é acompanhada pelo desenvolvimento de um esquema o qual tenta expressar a distribuição diferencial de cada um dos dados, conforme critérios específicos de decisão. A padronização tem por finalidade expressar os indicadores em unidades comparáveis entre si.

Entre os critérios de padronização ou uniformização de indicadores, podem ser mencionados as funçōes lineares (contínuas ou segmentadas), funções não li- neares (contínuas ou segmentadas) e o método de normalização. Este último método é muito empregado por órgãos ambientais sendo empregado na confecção, por exemplo, do IDH (Índice de Desenvolvimento Humano) das Nações Unidas.

A metodologia utilizada neste trabalho para a padronização dos indicadores foi baseada no método da normalização (Ott, 1978).

O procedimento matemático para calcular os indicadores padronizados ini- 
cia-se com a unificação das escalas. $\mathrm{Ou}$ seja, para qualquer dimensão, os indicadores de cada município são transformados em uma escala variável de 0 a 1 , através da expressão 1.

$\mathrm{Na}$ tabela 1 são apresentados os valores máximos e mínimos para os indicadores formadores do IIRSP para os municípios amostrados na regiāo $R$ e faixa populacional $P$. Quando os limites de aplicação forem ultrapassados (indicadores máximos e mínimos) deverá ser realizado novamente o procedimento não paramétrico de Kendall. O resultado é um indicador padronizado variando entre 0 e 1 , o qual será a base de cálculo para a construção do índice específico.

\section{RESULTADOS E DISCUSSÃO}

Inicialmente irá se abordar a formulação das equaçōes que formarão o Índice de Impacto dos Resíduos Sólidos Urbanos na Saúde Pública (IIRSP) e, posteriormente, se analisarão os resultados de seu emprego com dados reais.

\section{Formulação das equações com dados existentes}

A Tabela 2 apresenta as equações obtidas por intermédio do uso de procedimentos não paramétricos de Kendall para o IIRSP (nível de significância dos testes estatísticos de 5\%), podendo ser utilizadas para calcular este índice para os diversos municípios do estado do Rio Grande do Sul de acordo com a região e faixa populacional. Para o uso dessas equações os indicadores devem, primeiramente, estar padronizados em função da expressão 1 .

Com relação às percentagens relativas de cada indicador na composição do IIRSP, verificou-se, para as regiōes e faixas populacionais do estado do Rio Grande do Sul, que:

- o indicador de cisticercose apresenta um valor bastante significativo na região sul do estado para a faixa populacional menor que 10.000 habitantes urbanos;

- o indicador toxoplasmose é importante na região nordeste do estado para a faixa populacional entre 10.000 e 100.000 habitantes;

- para os indicadores de orçamento, deficiência de coleta e de tratamento/disposição final a contribuição ao índice é de menor relevância em todas; $\mathrm{e}$

- os altos valores dos coeficientes dos indicadores $\mathrm{IND}_{1}, \mathrm{IND}_{4}$ e IND , pro-

$I N D_{n ; X ; R ; P}=\frac{\left(V_{n ; X ; R ; P}-V \min _{n ; X ; R ; P}\right)}{\left(V \max _{n ; X ; R ; P}-V \min _{n ; X ; R ; P}\right)}$

onde:

$\mathrm{IND}_{\mathrm{n} ; \mathrm{X}: \mathrm{R}: \mathrm{P}}=$ Indicador padronizado $n$ no município $X$ da região $R$ e faixa populacional $P$;

$\mathrm{V}_{\mathrm{n} ; \mathrm{X} ; \mathrm{R} ; \mathrm{P}}=$ Valor do Indicador $n$ no município $X$ da região $R$ e faixa populacional $P$;

$V_{\text {min }}{ }_{\mathrm{n}: \mathrm{X}: \mathrm{R}: \mathrm{P}}=$ Valor mínimo do Indicador $n$ na região $R$ e faixa populacional $P$; e

$\operatorname{Vmax}_{\mathrm{n} ; \mathrm{X} ; \mathrm{R} ; \mathrm{P}}=$ Valor máximo do Indicador $n$ na região $R$ e faixa populacional $P$.

Tabela I - Valores máximos e mínimos dos indicadores formadores do Índice de Impacto dos Resíduos Sólidos Urbanos na Saúde Pública (IIRSP) para os municípios amostrados por região e faixa populacional

\begin{tabular}{cccccccccc}
\hline Região/Faixa Populacional & $\mathrm{IND}_{1}$ & $\mathrm{IND}_{2}$ & $\mathrm{IND}_{3}$ & $\mathrm{IND}_{4}$ & $\mathrm{IND}_{5}$ & $\mathrm{IND}_{7}$ & $\mathrm{IND}_{14}$ & $\mathrm{IND}_{41}$ \\
\hline Valores máximos & dos indicadores & formadores & do IIRSP \\
Mista/Faixa C & 0 & 15 & 0 & 2 & 0 & 6,58 & 45 & 50 \\
Nordeste/Faixa A & 0 & 1 & 0 & 5 & 0 & 9,50 & 42 & 100 \\
Nordeste/Faixa B & 0 & 9 & 0 & 5 & 0 & 12 & 20 & 100 \\
Sul/Faixa A & 2 & 5 & 0 & 1 & 0 & 5,70 & 40 & 40 \\
Sul/Faixa B & 1 & 116 & 0 & 44 & 3 & 12,37 & 40 & 40 \\
Norte/Faixa A & 1 & 12 & 67 & 7 & 2 & 5,38 & 80 & 100 \\
Norte/Faixa B & 0 & 18 & 0 & 24 & 0 & 10 & 30 & 100 \\
& & Valores mínimos dos indicadores formadores do IIRSP \\
Mista/Faixa C & 0 & 1 & 0 & 0 & 0 & 1,55 & 0 & 0 \\
Nordeste/Faixa A & 0 & 0 & 0 & 0 & 0 & 0,10 & 0 & 0 \\
Nordeste/Faixa B & 0 & 0 & 0 & 0 & 0 & 0,84 & 0 & 0 \\
Sul/Faixa A & 0 & 0 & 0 & 0 & 0 & 0,12 & 0 & 0 \\
Sul/Faixa B & 0 & 0 & 0 & 0 & 0 & 0,35 & 0 & 0 \\
Norte/Faixa A & 0 & 0 & 0 & 0 & 0 & 0,10 & 0 & 0 \\
Norte/Faixa B & 0 & 0 & 0 & 0 & 0 & 0,60 & 0 & 0 \\
\hline
\end{tabular}

Obs.: IND 1 - Indicador de Cisticercose; $\mathrm{IND}_{2}$ - Indicador de Leptospirose; $\mathrm{IND}_{3}$ Indicador de Teníase; $\mathrm{IND}_{4}$ - Indicador de Toxoplasmose; IND 5 - Indicador de Triquinose; $\mathrm{IND}_{7}$ - Indicador do Orçamento dos SLUs, $\mathrm{IND}_{14}$ - Indicador de Déficit de Coleta; $\mathrm{IND}_{41}$ - Indicador de Déficit de Tratamento/Disposição Final; Faixa A: Municípios de pequeno porte, população urbana menor que 10.000 hab.; Faixa B: Municípios de médio porte, população urbana entre 10.000 e 100.000 hab.; Faixa C: Municípios de grande porte, população urbana maior que 100.000 hab.

vavelmente, devem estar relacionados com a adoção do número total de casos das doenças informadas pelos municípios, pois não há como saber a verdadeira contribuição dos SLUs na ocorrência destas.

\section{Aplicação do índice de impacto dos resíduos sólidos urbanos na saúde pública}

Neste item serão analisados os resultados obtidos na aplicação do IIRSP aos municípios das regiōes sul, norte, nordeste e mista do Estado do Rio Grande do Sul, sendo que em cada região e faixa populacional o número de municípios amostrados foi: região nordeste/faixa A (23 municípios); região norte/faixa A (90 municípios), regiāo sul/faixa A (22 municípios), região nordeste/faixa B (23 municípios), regiāo Norte/faixa B (25 municípios), região Sul/faixa B (15 municípios) e região mista/faixa $\mathrm{C}$ (9 municípios).

a) Região Sul - Faixa populacional menor que 10.000 habitantes

O IIRSP para estes municípios apresentou uma variação entre $5 \%$ a 77,49\% com um valor médio de $67,99 \%$. A maioria dos municípios mostrou uma deficiência de cerca de $100 \%$ no tratamento/disposição final, sendo que a média do déficit, nestes municípios, foi de $91,36 \%$. Existe também um déficit de coleta de resíduos em $27 \%$ dos municípios.

O município de Tavares obteve o maior valor do IIRSP $(77,49 \%)$, tendo 
Tabela 2 - Exemplo das equações do Índice de Impacto dos Resíduos Sólidos Urbanos na Saúde Pública (IIRSP) para os Municípios da Região R e Faixa Populacional P do Estado do Rio Grande do Sul

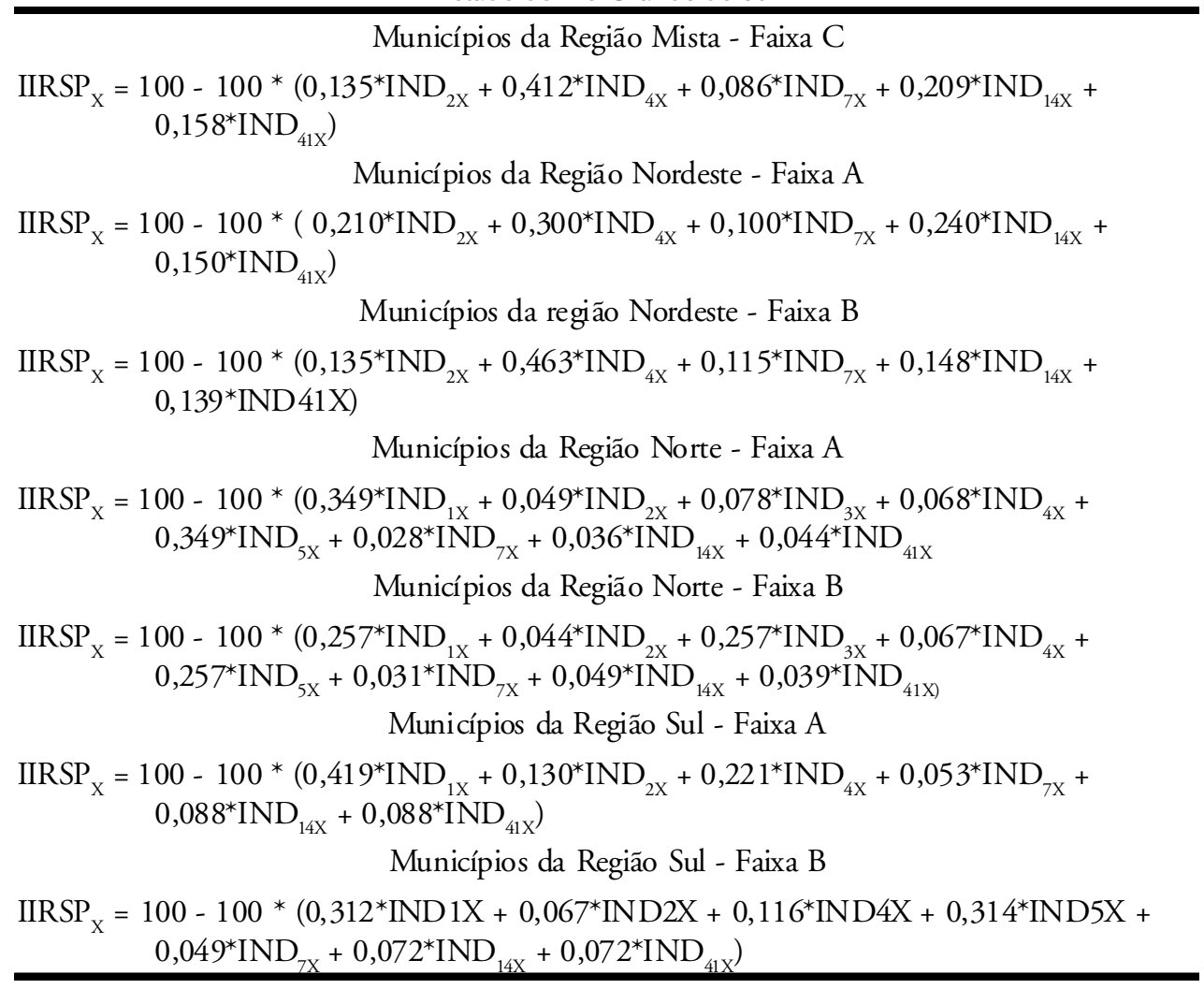

Obs.: IND - Indicador de cisticercose; $\mathrm{IND}_{2}$ - Indicador de leptospirose; $\mathrm{IND}_{3}$ - Indicador de teníase; $\mathrm{IND}_{4}$ - Indicador de toxoplasmose; $\mathrm{IND}_{5}$ - Indicador de triquinose; $\mathrm{IND}_{7}$ - Indicador de orçamento destinado aos SLUs, IND ${ }_{14}$ - Indicador de déficit de coleta; $\mathrm{IND}_{41}$ - Indicador de déficit de tratamento/disposição final eX - Refere-se ao município. Os indicadores das equaçōes devem estar padronizados; Faixa A - População menor que 10.000 hab.; Faixa B - População entre 10.000 e 100.000 hab.; Faixa C - População maior que 100.000 hab..

um déficit de tratamento/disposição final de $80 \%$; no entanto, a coleta dos resíduos atinge $100 \%$ da população urbana e não há casos das doenças consideradas neste índice. No município de Pântano Grande, o IIRSP foi de 5\%, apresentando um déficit de tratamento/disposição final de $100 \%$, de coleta de $30 \%$, dois casos de cisticercose, um caso de leptospirose e um caso de toxoplasmose, justificando o valor do índice.

Dados de cerca de $77 \%$ dos municípios apontaram um IIRSP inferior a $40 \%$. Nos municípios de pequeno porte do estado, normalmente, não há um controle sistemático com relação às doenças avaliadas por este índice.

b) Região Sul - Faixa populacional entre 10.000 e 100.000 habitantes

Em sete municípios, o IIRSP apresentou valores superiores a $60 \%$. O município de Charqueadas induziu a um IIRSP de 40,58\% devido, principalmen- te, à deficiência na coleta e no tratamento/disposição final em cerca de $40 \%$ e ocorrência de cinco casos de leptospirose.

Nos municípios de Rosário do Sul (IIRSP $=5 \%$ ) e Canguçu (IIRSP = 6,42\%), além da deficiência de tratamento/disposição final de $100 \%$, existe um déficit de coleta (de 20 e de 5\%, respectivamente). Em Rosário do Sul foram notificados casos de cisticercose (1), de leptospirose (116) e de toxoplasmose (2). Em Canguçu, os casos notificados eram referentes à leptospirose (15), à toxoplasmose (44) e à triquinose (3). Demonstra-se uma forte correlação entre a deficiência de coleta e de tratamento/disposição final e doenças, nesta região e faixa populacional.

c) Regiāo Mista - Faixa populacional maior que 100.000 habitantes

O IIRSP apresentou um valor médio de $64,93 \%$, sendo que o menor valor foi encontrado no município de Canoas $($ IIRSP $=47,76)$, devido, principalmen- te, à existência de casos de leptospirose (10), de toxoplasmose (2) e de um déficit de tratamento/disposição final de $2 \%$. Entretanto, o que influiu com maior peso no valor do índice foram os casos de leptospirose.

Em Viamão, o IIRSP foi de 49,51\%, devido a doze casos de leptospirose, déficit de coleta de $45 \%$ e de tratamento/disposição final de $50 \%$. Os municípios de Novo Hamburgo, Passo Fundo, São Leopoldo, Sapucaia do Sul e Uruguaiana tiveram um IIRSP maior que $60 \%$. No entanto, em Novo Hamburgo há um déficit de coleta e de tratamento/disposição final de 5\% e foram notificados dez casos de leptospirose. Passo Fundo tem um déficit de tratamento/disposição final de $10 \%$ e com dois casos de leptospirose. Uruguaiana notificou um caso de leptospirose. Mesmo assim, o município de Uruguaiana apresentou um IIRSP de $85,37 \%$. O indicador leptospirose é res- 
ponsável em todos municípios pela redução do valor do IIRSP.

d) Região Nordeste - Faixa populacional menor que 10.000 habitantes

Os municípios da região nordeste induziram a um SIIRSP entre 31,10\% (Feliz) a $81,01 \%$ (Presidente Lucena) com média de 63,60\%. Há um déficit médio de tratamento/disposição final nos municípios de 76,74\%. Alguns municípios apresentam casos de leptospirose e de toxoplasmose. O município de Feliz detectou dois casos de toxoplasmose e uma deficiência de tratamento/disposição final da ordem de $100 \%$, de coleta de $20 \%$ sendo o percentual do orçamento destinado aos SLUs inferior a média regional. Dois municípios apresentaram valores do índice acima de $80 \%$.

e) Regiāo Nordeste - Faixa populacional entre 10.000 e 100.000 habitantes

A faixa de variação do IIRSP foi de $20,14 \%$ a $82,56 \%$, com média de $67,06 \%$. Verifica-se deficiência de tratamento/disposição final em $83 \%$ dos municípios e $52 \%$ com déficit de coleta (porém estes valores na maioria dos municípios é inferior a 5\%). Foram notificados casos de leptospirose em muitos municípios.

Eldorado e Parobé apresentaram um IIRSP de $23,18 \%$ e $20,14 \%$, respectivamente. Em Eldorado há um déficit de tratamento/disposição final de 100\%. Neste município ocorreram nove casos de leptospirose, cinco casos de toxoplasmose e o orçamento destinado aos SLUs é baixo em relação aos outros municípios. Parobé apresenta um déficit de tratamento/disposição final de $90 \%$ e de coleta de $11 \%$, dois casos de leptospirose e um caso de toxoplasmose.

f) Região Norte - Faixa populacional menor que 10.000 habitantes

Nesta região, cerca de $34 \%$ dos municípios obtiveram um IIRSP entre 60\% e 80\%. Entretanto, em 2\% dos municípios, o valor do IIRSP foi inferior a 20\%. Na maioria dos municípios desta região e faixa populacional, há deficiência de tratamento/disposição final de $100 \%$, alguns com déficit na coleta dos resíduos, casos de leptospirose, casos de teníase, casos de toxoplasmose e baixa percentagem do orçamento destinado aos SLUs.

g) Região Norte - Faixa populacional populacional entre $10.000 \mathrm{e}$ 100.000 habitantes

Cerca de 32\% dos municípios possuem um IIRSP entre $20 \%$ e $40 \%$ e em
68\% dos municípios este valor foi inferior a $20 \%$. É significativa a deficiência de tratamento/disposição final nos municípios amostrados, bem como, casos de leptospirose e toxoplasmose.

Em Três de Maio (IIRSP = 5\%) há um déficit de tratamento/disposição final de $100 \%$, quatro casos de leptospirose e 24 de toxoplasmose. Santa Cruz do Sul (IIRSP $=7,86 \%$ ) possui uma deficiência de tratamento/disposição final de $15 \%$, 18 casos de leptospirose e cinco de toxoplasmose. Santa Rosa (IIRSP = 3\%) tem uma deficiência de tratamento/disposição final de $100 \%$, sete casos de leptospirose e oito de toxoplasmose.

\section{CONCLUSÕES}

O índice IIRSP desenvolvido ajuda a espelhar a relação existente entre as características das comunidades regionais relacionadas aos resíduos sólidos e à saúde pública. Apesar de fazer parte de um índice geral, o IIRSP e suas equações desenvolvidas serviram para preencher lacunas de dados em comunidades de características econômicas e populacionais semelhantes às empregadas.

O IIRSP permite avaliar setores particulares da limpeza urbana quando se necessita tomar decisóes gerenciais em alguma das etapas dos serviços de limpeza urbana.

As equaçôes obtidas para calcular o IIRSP demonstraram que os indicadores de doenças representam, estatisticamente, a maior fração do índice. Esta metodologia pode e foi empregada para avaliar a contribuição de outros aspectos da eficiência dos serviços de limpeza urbana em índice geral de qualidade dos sistemas de gerenciamento de resíduos sólidos.

Especificamente, verifica-se a necessidade de realizar estudos epidemiológicos mais aprofundados para separar, estatisticamente, os efeitos da ocorrência de doenças por outras fontes concorrentes dos resíduos sólidos urbanos. Para isso, espera-se que as secretarias de saúde tenham condiçôes de atualizar e aprimorar seus bancos de dados.

\section{AGRADECIMENTOS}

Os autores agradecem os dados gentilmente cedidos pelas Secretarias de Saúde Municipais e pela Secretaria de Saúde do Estado do Rio Grande do Sul. Bolsas da CAPES e do CNPq e recursos da Secretaria de Desenvolvimento Urbano (SEDU), Gabinete da Presidência da
República, possibilitaram a pesquisa de campo e a conclusão da tese de doutoramento.

\section{REFERÊNCIAS}

ADRIAANSE, A. Environmental policy performance indicators. General of Environmental Dutch Ministry of Housing. 35p, 1993.

BAKKES, J. A. et al. An overview of environmental indicators: state of the art perspectives. Environment Assessment Technical Reports, RIVM in co-operation with the University of Cambridge, UNEP-RIUM, 1994 apud WINOGRAD, Manuel. Capacidad institutional para la producción y análisis de datos ambientales em los paises de América Latina y el Caribe. Cali: CIAT. f.26. 1995.

DE LUCA, S. J. (coord.). Avaliação dos serviços de limpeza urbana no Brasil: relatório final. Porto Alegre: Instituto de Pesquisas Hidráulicas da UFRGS, [Brasília]: Secretaria Especial de Desenvolvimento Urbano. 1v. 1999.

DEUS, A. B. S. de. Gerenciamento de serviços de limpeza urbana: Avaliação por indicadores e indices. Tese de Doutorado. Universidade Federal do Rio Grande do Sul. Instituto de Pesquisas Hidráulicas. Porto Alegre, BR-RS, 253 f.: il. Orien: De Luca, Sérgio João. 2000.

OLIVEIRA, N. et al. Dinâmica da urbanização no RS: temas e tendências. 2. ed. Porto Alegre: FEE. 271p. (Documentos FEE). 1998.

OTT, W. R. Environmental indices: theory and practice. Ann Arbor: Ann Arbor Science. 371p. 1978.

SIEGEL, S. Estatística não-paramétrica para as ciências do comportamento. New York: McGrawHill. 1981.

UNEP. The role of indicators in decision-making. Paris. 1995.

Endereço para correspondência:

Ana Beatris Souza de Deus Universidade de Caxias do Sul Instituto de Saneamento Ambiental Rua Francisco Getúlio Vargas, I I 30 9500 I-970 - Caxias do Sul - RS Brasil

Tel.: (5 I) 99 I 6-9256

E-mail:absdeus@terra.com.br 